Monatsschr Kinderheilkd

1999•147:56-59 (C) Springer-Verlag 1999

Redaktion:

P.Emmrich, München

K. Kruse, Lübeck

\title{
Rekombinanter Borrelia-burgdorferi-Impfstoff mit Kapsellipoprotein A und Adjuvanz
}

AC Stern et al. and the Lyme Disease Vaccine Study Group (1998) Vaccination against Lyme disease with recombinant Borrelia Burgdorferi outer-surface lipoprotein A with adjuvant. N Engl J Med 339:209

\section{Ergebnisse}

erkranken, ist in den Gebieten hoch, in denen die Krankheit endemisch vorkommt. Die Entwicklung eines sicheren und effektiven Impfstoffs ist daher von großer Bedeutung.

\section{Methoden}

Es wurde eine multizentrische, doppelblinde randomisierte Impfstudie an 10.936 Probanden durchgeführt, die alle zum Zeitpunkt der Studie in verschiedenen Gebieten der USA lebten, in denen die Lyme-Borreliose endemisch vorkommt. Die Studienteilnehmer erhielten jeweils zu Beginn der Studie sowie 1 und 12 Monate später entweder einen rekombinanten Borrelia-burgdorferi-Impfstoff, der Kapsellipoprotein A (30 $\mu \mathrm{g}$ OspA) und ein Adjuvanz enthielt, oder ein Placebo. Bei vermuteter Borreliose wurde entweder eine Kultur der Hautläsionen, eine PCR oder ein serologischer Nachweis durchgeführt. Um eine asymptomatische Infektion nachzuweisen, erfolgte 12 und 24 Monate nach Studienbeginn eine serologische Untersuchung.
Nach jeweils 2 Injektionen erkrankten innerhalb des ersten Jahres 22 Probanden der Impfgruppe und 43 der Placebogruppe an einer gesicherten Borreliose ( $p$ 0,009). Dies entspricht einer Effizienz der Impfung von $49 \%$.

Nach der 3. Impfung zeigten 16 Impflinge und 66 Probanden der Placebogruppe eindeutige Hinweise auf eine Borreliose $(p<0,001)$. Die Effizienz lag somit bei $76 \%$. Im Hinblick auf die Prävention einer asymptomatischen Infektion lag die Effizienz bei $83 \%$ im 1 . und bei $100 \%$ im 2. Jahr.

Die Injektion des Impfstoffes war assoziiert mit leichten bis mäßgiggradigen lokalen $(26,8 \%)$ oder systemischen $(19,4 \%)$ Nebenwirkungen, die im Mittel 3 Tage anhielten.

\section{Schlußfolgerungen}

Drei Injektionen eines Lyme-BorrelienImpfstoffes waren in der Lage, in den meisten Fällen eine B. burgdorferi-Erkrankung und in allen Fällen eine asympotmatische Infektion zu verhindem.

\section{$\checkmark$ Kommentar}

Im genannten Heft des New Engl J Med erscheint eine weitere multizentrische Studie, in der der gleiche Impfstoff bei gleichem Studiendesign an einem in etwa gleich großen Kollektiv getestet wurde (Signal et al, pp 216). Die Effizienz lag mit $68 \%$ t nach 2 und bei $92 \%$ nach 3 Dosen höher als in der anderen Studie.

In einem Editorial weisen Steigbigel und Benach (S.263) auf folgende noch ungeklärten Fragen hin:

Die Häufigkeit einer Lyme-Borreliose ist besonders hoch im Kindesalter. In die vorliegenden Studien wurden jedoch keine Probanden im Kindesalter eingeschlossen.

Die Dauer des Impfschutzes ist noch ungeklärt, so daß sich die Frage nach Zeitpunkt und Zahl von Booster-Injektionen stellt.

D Das im Impfstoff enthaltende Kapsellipoprotein A (OspA) von B. burgdorferi ist in den USA verbreitet, in anderen Ländern aber möglicherweise heterogen.

D Die Studien belegen, daß die neue Vakzine wirksam ist und zur Prävention der Lyme-Borreliose in Regionen, in denen die Erkrankung endemisch vorkommt, eingesetzt werden kann.

Da das Genom von B. burgdorferi vor kurzem sequenziert wurde, wird möglicherweise in Bälde ein noch wirksamerer Impfstoff verfügbar sein. 